\title{
The Role of Microstructure of Highly Purified Beta-Tricalcium Phosphate for Osteoinduction in Canine Dorsal Muscles
}

\author{
Takashi Ariizumi $^{{ }^{*}}$, Akira Ogose ${ }^{1}$, Naoki Kondo ${ }^{1}$, Hiroyuki Kawashima ${ }^{1}$, Tetsuo Hotta ${ }^{1}$, \\ Naoko Kudo ${ }^{2}$, Makiko Hoshino ${ }^{1}$, Hikaru Inoue ${ }^{3}$, Hiroyuki Irie ${ }^{4}$, Naoto Endo ${ }^{1}$ \\ ${ }^{1}$ Division of Orthopedic Surgery, Niigata University Graduate School of Medical and Dental Sciences, Niigata, Japan; ${ }^{2}$ Division of \\ Orthopedic Surgery, Niigata Prefectural Central Hospital, Niigata, Japan; ${ }^{3}$ Development Department, Olympus Terumo Biomaterial \\ Corp., Tokyo, Japan; ${ }^{4}$ Osteopharma Inc., Osaka, Japan. \\ Email: ${ }^{*}$ arii2075@med.niigata-u.ac.jp, aogose@med.niigata-u.ac.jp
}

Received January $13^{\text {th }}, 2013$; revised March $7^{\text {th }}, 2013$; accepted April $9^{\text {th }}, 2013$

Copyright (C) 2013 Takashi Ariizumi et al. This is an open access article distributed under the Creative Commons Attribution License, which permits unrestricted use, distribution, and reproduction in any medium, provided the original work is properly cited.

\begin{abstract}
Porous $\beta$-tricalcium phosphate (TCP) displays osteoinductivity in certain animals in the absence of osteoinductive agents. We evaluated whether the microstructure may be an important determinant of osteoinduction, and also investigated how bone formation was promoted using $\beta$-TCP combined with bone marrow aspirates. We prepared two types of $\beta$-TCP, namely, $\beta$-TCP A, which possessed interconnected macropores and micropores, and $\beta$-TCP $\mathrm{B}$, which possessed macropores but had less detectable micropores. These were implanted with or without marrow in canine muscles. Bone formation and the resorption of each $\beta$-TCP implant were evaluated histologically. Newly formed bone began to appear at day 42 in the implants of $\beta$-TCP A alone, but the implants of $\beta$-TCP B alone did not show any bone formation by day 42. Meanwhile, bone formation was already evident on day 14 by loading with bone marrow aspirates with or without micropores. By immunohistochemistry, the number of cathepsin K-positive cells (osteoclasts) increased as time passed in the implants of $\beta$-TCP A alone, while the number of the osteoclasts did not change obviously in the implants of $\beta$-TCP B alone from day 14 to 56 . Reticular fibrils were evident within the $\beta$-TCP A, and were barely observed in the $\beta$-TCP B in the silver impregnation. The present result would bring about the possible role to enhance the importance of the surface microstructure for the better osteoinductivity. Our findings suggest that the combination of porous $\beta$-TCP and bone marrow facilitates bone formation.
\end{abstract}

Keywords: Osteoinduction; Beta-Tricalcium Phosphate; Micropore; Bioresorption; Osteoclast

\section{Introduction}

Recently, bioactive ceramics such as hydroxyapatite and calcium phosphate have been widely used to fill bone defects after either trauma or a surgical resection of bone tumors [1-5]. We have focused our attention on porous $\beta$-tricalcium phosphate ( $\beta$-TCP) [3-7]. Numerous studies have demonstrated that porous $\beta$-TCP has good biocompatibility and bioactivity: furthermore, it is absorbed and replaced by autologous bone [3-8]. We previously reported that $\beta$-TCP enables osteoconduction in the rat femoral condyle and human bone in the absence of additional osteoinductive agents $[3,6]$. Meanwhile, osteoin-

"Corresponding author. ductive properties are required for the large bony defects [9]. Porous $\beta$-TCP displays osteoinductivity in certain animals [10,11]. Kondo et al. observed osteoinduction with highly purified $\beta$-TCP in canine dorsal muscles and suggest that the micropores on the macropore surface are a critical factor in the process of osteoinduction [7]. In this study, we investigated whether the microstructure may be an important determinant of osteoinduction. Furthermore, bone marrow has a good osteogenic ability when combined with porous ceramics, and has the clinical merits of being autologous and intraoperatively available, with harvesting done by a simple, safe aspiration [12]. We also investigated how bone formation was promoted using $\beta$-TCP combined with bone marrow aspirates. 


\section{Experimental and Methods}

\subsection{Preparation of $\beta$-TCP}

We prepared two types of $\beta$-TCP; $\beta$-TCP A, which possessed interconnected macropores of $100-500 \mu \mathrm{m}$ and micropores of less than $5 \mu \mathrm{m}$ (Figures 1(A) and (C)), and $\beta$-TCP $\mathrm{B}$, which also possessed macropores but had less detectable micropores on the surface of the macropores (Figures 1(B) and (D)). Beta-TCP A and B were ob- tained from Olympus Terumo Biomaterials Corp. (Tokyo, Japan). Fine $\beta$-TCP powder was mechanochemically synthesized by wet milling. $\mathrm{CaHPO}_{4} \cdot \mathrm{H}_{2} \mathrm{O}$ and $\mathrm{CaCO}_{3}$ at a molar ratio of $2: 1$ were mixed into a slurry with pure water and particles of zirconium in a pot mill for 24 hours, and dried at $80^{\circ} \mathrm{C}$, leading to the formation of cal- cium-deficient hydroxyapatite. This crystalline solid was converted to $\beta$-TCP by calcination at $750^{\circ} \mathrm{C}$ for 1 hour. A porous $\beta$-TCP block was obtained upon sintering of the $\beta$-TCP powder, and this block was classified by an as- sessment of the surface area and pore-size distribution. The $\beta$-TCP A blocks displayed $75 \%$ porosity and $\beta$-TCP B blocks showed $50 \%$ porosity. The surface area, which was measured by the Brunauer-EmmettTeller method, was $1.4 \mathrm{~m}^{2} / \mathrm{g}$. Five-millimeter cubic blocks of $\beta$-TCP were used in the study.

\subsection{Animal Model and Tissue Preparation}

A total of four twelve-month-old female beagle dogs were used in this study. The paravertebral muscle in each dog was exposed in sterile conditions under general anesthesia. A total of four experimental groups were prepared as follows: group $1, \beta$-TCP A alone; group $2, \beta$ TCP $\mathrm{B}$ alone; group $3, \beta$-TCP A loaded with bone marrow aspirates; group $4, \beta$-TCP B loaded with bone marrow aspirates. Bone marrow was obtained by aspiration from the iliac bone using a syringe. In group 1 and $2, \beta$ TCP blocks were implanted into the muscles directly. In group 3 and 4, $\beta$-TCP blocks were infiltrated with bone marrow aspirates, and were implanted into the muscles 30 minutes after infiltration. All animal experiments were conducted according to the "Guidelines for Animal Experimentation" of Olympus Corporation and were approved by the ethical committee of Olympus medical systems.

On post-operative days $14,28,42,56$ and 112 , the dogs were anesthetized and the implanted $\beta$-TCP blocks were extracted and immersed in $4 \%$ paraformaldehyde for 5 - 7 days. The $\beta$-TCP blocks were decalcified with etylenediaminetetraacetic acid and embedded with paraffin and evaluated histologically.

\subsection{Cathepsin K Immunohistochemistry}

Cathepsin $\mathrm{K}$ is a specific collagenase synthesized by os- teoclasts, and it is essential for bone resorption [13]; there- fore, cathepsin $\mathrm{K}$ immunohistochemistry was performed to detect active osteoclasts. The paraffin embedded-spe- cimens were sectioned at $4 \mu \mathrm{m}$ and immunostained by cathepsin $\mathrm{K}$ as previously described [7].

\subsection{Reticulin Silver Impregnation}

The implants were stained by silver impregnation to detect collagen and reticular fibers, as previously described [14].

\subsection{Statistical Analysis}

Statistical analyses were performed using the SPSS 14.0J software package for Windows with the Tukey test (either one-way ANOVA or a post hoc test) used for the ratio of bone formation at each time point. A value of $\mathrm{P}<$ 0.05 was considered to indicate a statistically significant difference.

\section{Results}

All implants in group 1 and 2 showed fibrovascular invasion into macropores. However, there was no evidence of any newly formed bone by days 14 (Figures 2(A) and (B)) or 28. Newly formed bone began to appear at day 42 in the implants of group1 (Figure 2(C)), and obvious bone formation was detected on days 56 and 112. The implants in group 2 did not show any bone formation by day 42 (Figure 2(D)) and newly formed bone was detected in only half of the implants on days 56 and 112. Meanwhile, bone formation was already evident on day 14 in the implants of group 3 and 4, and newly formed bone of group 3 was detected more frequently and widely than that of group 4 (Figures 2(E) and (F)). Table 1 sum- marized the ratio of bone formation at each time

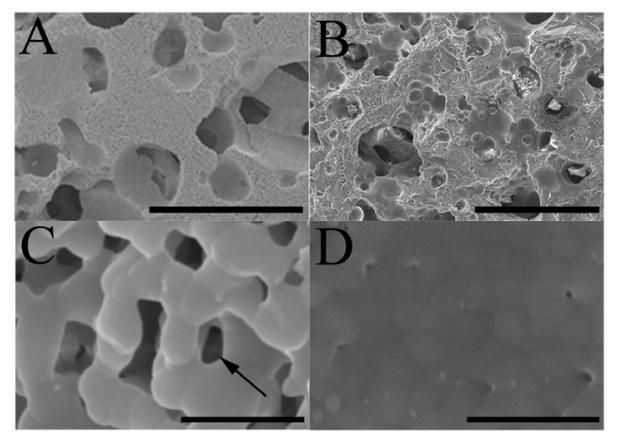

Figure 1. The microstructure of the two types of $\beta$-TCP by scanning electron microscope; Beta-TCP A possesses interconnected macropores of $100-500 \mu \mathrm{m}(\mathrm{A})$ and numerous micropores (arrows) of less than $5 \mu \mathrm{m}$ on the surface of macropores (C), while $\beta$-TCP B also possessed interconnected macropores (B) but had less detectable micropores (D) on the surface of the macropores. Bar: $500 \mu \mathrm{m}$ (A, B); 5 $\mu \mathrm{m}(\mathrm{C}, \mathrm{D})$. 


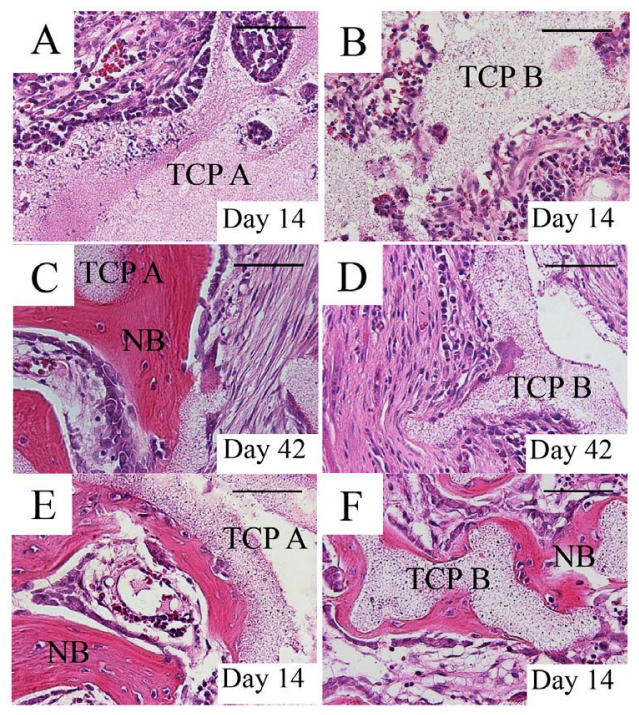

Figure 2. Histological sections stained with hematoxylin and eosin in the $\beta$-TCP implants; All $\beta$-TCP A and B implants without marrow showed fibrovascular invasion into macropores; however, there was no evidence of any newly formed bone on day $14(\mathrm{~A}, \mathrm{~B})$. On day 42, bone began to appear in the group $1(\mathrm{C})$, while the implants of group 2 did not show any bone formation (D). In the implants group 3 and 4, bone formation occurred on day 14, and newly formed bone was detected in almost all $\beta$-TCP A implants (E), while bone formation was infrequent and new bone was only locally formed in $\beta$-TCP B implants (F). Group 1: $\beta$-TCP A alone; Group 2: $\beta$-TCP B alone; Group 3: $\beta$-TCP A loaded with bone marrow aspirates; Group 4: $\beta$-TCP B loaded with bone marrow aspirates; TCP, $\beta$-tricalcium phosphate; NB, newly formed bone. Bar: $100 \mu \mathrm{m}$.

Table 1. The ratio of bone formation at each time point.

\begin{tabular}{ccccc}
\hline $\begin{array}{c}\text { Osteogenic implants number/ } \\
\text { total implants number }\end{array}$ & $\begin{array}{c}\text { Group } \\
1\end{array}$ & $\begin{array}{c}\text { Group } \\
2\end{array}$ & $\begin{array}{c}\text { Group } \\
3\end{array}$ & $\begin{array}{c}\text { Group } \\
4\end{array}$ \\
\hline Day 14 & $0 / 9$ & $0 / 4$ & $4 / 5$ & $1 / 5$ \\
Day 28 & $0 / 9$ & $0 / 4$ & $5 / 5$ & $1 / 5$ \\
Day 42 & $1 / 5$ & $0 / 5$ & $5 / 5$ & $3 / 5$ \\
Day 56 & $5 / 7$ & $3 / 7$ & $2 / 2$ & $2 / 7$ \\
Day 112 & $12 / 13$ & $2 / 3$ & $3 / 3$ & $2 / 3$ \\
\hline
\end{tabular}

Group 1: $\beta$-TCP A alone; Group 2: $\beta$-TCP B alone; Group 3: $\beta$-TCP A loaded with bone marrow aspirates; Group 4 : $\beta$-TCP B loaded with bone marrow aspirates.

point in the four groups. But these differences were not statisti- cally significant.

By immunohistochemistry, only a few cathepsin K-positive cells (osteoclasts) were observed in the peripheral region of the implanted area of both group 1 and 2 on day 14. The number of osteoclasts increased as time passed in the implants of group 1 (Figure 3(A)), while the number of the osteoclasts did not change obviously in the implants of group 2 from day 14 to 56 (Figure 3(B)).

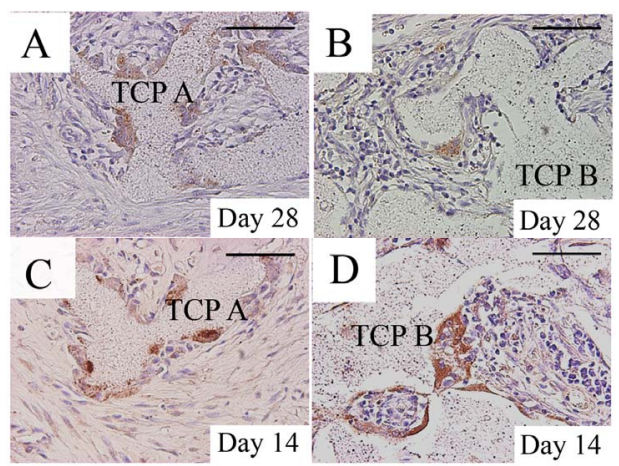

Figure 3. Evidence of osteoclasts by Cathepsin K immunohistochemistry in the $\beta$-TCP implants; The number of cathepsin K-positive multinucleated cells (osteoclasts) had increased by day 28 in the implants of group 1 (A), while only a few osteoclasts were observed in the peripheral region of the implanted area in the implants of group 2 (B). Numerous osteoclasts were observed in the peripheral region in the implants of group 3 on day 14 (C). Osteoclasts were also detected in the implants of group 4 (D), although the number of osteoclasts was less than that of group 3. Group 1: $\beta$-TCP A alone; Group 2: $\beta$-TCP B alone; Group 3: $\beta$-TCP $A$ loaded with bone marrow aspirates; Group 4: $\beta$-TCP B loaded with bone marrow aspirates TCP, $\beta$-tricalcium phosphate; NB, newly formed bone. Bar: $100 \mu \mathrm{m}$.

Numerous osteoclasts were observed in the implants of group 3 on day 14 (Figure 3(C)), and osteoclasts were observed in the central region of the implanted area as well as in the peripheral region from days 28 to 112 . Numerous osteoclasts were also detected in the implants of group 4 from day 14 (Figure 3(D)) to 112, but the number of these osteoclasts was less than that of group 3 .

Silver impregnation demonstrated the presence of reticular fibrils within the implants of group 1 or 3 (Figure 4(A)), while reticular fibrils were barely observed in the implants of group 2 or 4 (Figure 4(B)).

\section{Discussion}

We investigated the promotion of bone formation using $\beta$-TCP loaded with bone marrow aspirates. Bone formation occurred at a high rate in group 3 from the early period. Additionally, bone formation was facilitated in $\beta$ TCP without micropores because newly formed bone was observed in the implants of group 4. It is well-known that ectopic bone formation is induced by marrow cells in porous calcium phosphate ceramics [15]. These findings suggest that bone marrow cells are important as a source of osteoinduction.

We also evaluated the osteoinductive capacity of two types of $\beta$-TCP that had the same chemical composition but different internal structures. The ratio of newly formed bone in group 2 tended to be much smaller than that in group 1. Although the statistical significance has never been demonstrated, the present result would bring 


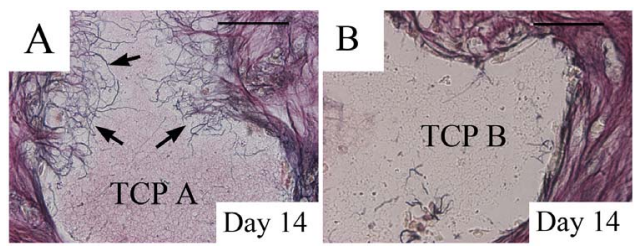

Figure 4. Silver impregnation of reticular fibrils; Reticular fibrils (arrows) were present within the $\beta$-TCP A on day 14 (A), while reticular fibrils were barely observed in the $\beta$ TCP B on day 14 (B). TCP, $\beta$-tricalcium phosphate; Bar: $100 \mu \mathrm{m}$.

about the possible role to enhance the importance of the microstructure for the better osteoinductivity. The importance of the microstructure has been analyzed in other bioactive ceramics $[16,17]$. These findings indicate that microstructure plays an important role in osteoinduction in various kinds of bioactive ceramics.

A histological analysis revealed that the number of osteoclasts increased earlier in group 1 than in group 2. Osteoclasts appeared before new bone was formed. Therefore, the microstructure of $\beta$-TCP may involve a progressive ostoclastic resorption. The importance of osteoclastic resorption in $\beta$-TCP has been reported previously [7, $18]$, and our results supports the previous studies and may demonstrate that the microstructure influences osteoclastic resorption before new bone is formed.

The reason that the microstructure is important for osteoinduction is unknown, but reticular fibrils were evident within the $\beta$-TCP A. The silver impregnation technique stains collagen, reticular fibers and elastic fibers [14]. Chazono et al. reported that the micropores in $\beta$ TCP ceramic may provide a suitable environment for collagen formation [19]. Ogose et al. revealed the intrusion of the collagen fibrils into the micropores of the $\beta$ TCP in the scanning electron microscope observation and suggested the protein absorption to the micropores [20]. The current study revealed the intrusion of the reticular fibrils including the collagen fibers into the micropores and provided the possible role that the micropores act as a storage space for proteins, including collagen fibers.

The main limitation of the current study is the use of the different porosity between the two types of $\beta$-TCP. The porosity of $\beta$-TCP scaffold can influence the ceramic resorption and bone formation [16,21]. In future, we need to incorporate the experiments to compare the two types of $\beta$-TCP with the same porosity but the different volume of micropores.

\section{Conclusion}

The present result would bring about the possible role to enhance the importance of the surface microstructure for the better osteoinductivity. The combination of porous $\beta$-TCP and bone marrow facilitates bone formation.

\section{Acknowledgements}

The authors would like to thank Yoshiaki Tanaka and Hideki Akazawa (Division of Orthopedic Surgery, Department of Regenerative and Transplant Medicine, Niigata University Graduate School of Medical and Dental Sciences).

\section{REFERENCES}

[1] T. Yamamoto, T. Onga, T. Marui and K. Mizuno, "Use of Hydroxyapatite to Fill Cavities after Excision of Benign Bone Tumours. Clinical Results," Journal of Bone and Joint Surgery, British Volume, Vol. 82, No. 3, 2000, pp. 1117-1120. doi:10.1302/0301-620X.82B8.11194

[2] A. Uchida, N. Araki, Y. Shinto, H. Yoshikawa, E. Kurisaki and K. Ono, "The Use of Calcium Hydroxyapatite Ceramic in Bone Tumour Surgery," Journal of Bone and Joint Surgery, British Volume, Vol. 72, No. 2, 1990, pp. 298-302.

[3] A. Ogose, T. Hotta, H. Hatano, H. Kawashima, K. Tokunaga, N. Endo and H. Umezu, "Histological Examination of Beta-Tricalcium Phosphate Graft in Human Femur," Journal of Biomedical Materials Research, Vol. 63, No. 5, 2002, pp. 601-604. doi:10.1002/jbm.10380

[4] A. Ogose, T. Hotta, H. Kawashima, N. Kondo, W. Gu, T. Kamura and N. Endo, "Comparison of Hydroxyapatite and Beta Tricalcium Phosphate as Bone Substitutes after Excision of Bone Tumors," Journal of Biomedical Materials Research, Part B, Applied Biomaterials, Vol. 72, No. 1, 2005, pp. 94-101. doi:10.1002/jbm.b.30136

[5] A. Ogose, N. Kondo, H. Umezu, T. Hotta, H. Kawashima, K. Tokunaga, T. Ito, N. Kudo, M. Hoshino, W. Gu and N. Endo, "Histological Assessment in Grafts of Highly Purified Beta-Tricalcium Phosphate (OSferion) in Human Bones," Biomaterials, Vol. 27, No. 8, 2006, pp. 15421549. doi:10.1016/j.biomaterials.2005.08.034

[6] N. Kondo, A. Ogose, K. Tokunaga, T. Ito, K. Arai, N. Kudo, H. Inoue, H. Irie and N. Endo, "Bone Formation and Resorption of Highly Purified Beta-Tricalcium Phosphate in the Rat Femoral Condyle," Biomaterials, Vol. 26, No. 28, 2005, pp. 5600-5608.

doi:10.1016/j.biomaterials.2005.02.026

[7] N. Kondo, A. Ogose, K. Tokunaga, H. Umezu, K. Arai, N. Kudo, M. Hoshino, H. Inoue, H. Irie, K. Kuroda, H. Mera and N. Endo, "Osteoinduction with Highly Purified BetaTricalcium Phosphate in Dog Dorsal Muscles and the Proliferation of Osteoclasts before Heterotopic Bone Formation," Biomaterials, Vol. 27, No. 25, 2006, pp. 44194427. doi:10.1016/j.biomaterials.2006.04.016

[8] K. Shiratori, K. Matsuzaka, Y. Koike, S. Murakami, M. Shimono and T. Inoue, "Bone Formation in Beta-Tricalcium Phosphate-Filled Bone Defects of the Rat Femur: Morphometric Analysis and Expression of Bone Related Protein mRNA," Biomedical Research, Vol. 26, No. 2, 2005, pp. 51-59. doi:10.2220/biomedres.26.51

[9] Y. Okubo, K. Bessho, K. Fujimura, Y. Konishi, K. Kusumoto, Y. Ogawa and T. Iizuka, "Osteoinduction by Recombinant Human Bone Morphogenetic Protein-2 at In- 
tramuscular, Intermuscular, Subcutaneous and Intrafatty Sites," International Journal of Oral and Maxillofacial Surgery, Vol. 29, No. 1, 2000, pp. 62-66. doi:10.1016/S0901-5027(00)80127-7

[10] D. Le Nihouannen, G. Daculsi, A. Saffarzadeh, O. Gauthier, S. Delplace, P. Pilet and P. Layrolle, "Ectopic Bone Formation by Microporous Calcium Phosphate Ceramic Particles in Sheep Muscles," Bone, Vol. 36, No. 6, 2005, pp. 1086-1093. doi:10.1016/j.bone.2005.02.017

[11] P. Habibovic, H. Yuan, C. M. van der Valk, G. Meijer, C. A. van Blitterswijk and K. de Groot, "3D Microenvironment as Essential Element for Osteoinduction by Biomaterials," Biomaterials, Vol. 26, No. 17, 2005, pp. 35653575. doi:10.1016/j.biomaterials.2004.09.056

[12] T. Yoshii, S. Sotome, I. Torigoe, A. Tsuchiya, H. Maehara, S. Ichinose and K. Shinomiya, "Fresh Bone Marrow Introduction into Porous Scaffolds Using a Simple LowPressure Loading Method for Effective Osteogenesis in a Rabbit Model," Journal of Orthopaedic Research, Vol. 27, No. 1, 2009, pp. 1-7. doi:10.1002/jor.20630

[13] B. R. Troen, "Cathepsin K Plays a Critical Role in the Degradation of Bone and Appears to Be a Limiting Step in Osteoclastic Bone Resorption," Drug News \& Perspectives, Vol. 17, No. 1, 2004, pp. 19-28. doi:10.1358/dnp.2004.17.1.829022

[14] T. Ushiki, "Collagen Fibers, Reticular Fibers and Elastic Fibers. A Comprehensive Understanding from a Morphological Viewpoint," Archives of Histology and Cytology, Vol. 65, No. 2, 2002, pp. 109-126. doi:10.1679/aohc.65.109

[15] H. Ohgushi, V. M. Goldberg and A. I. Caplan, "Heterotopic Osteogenesis in Porous Ceramics Induced by Marrow Cells," Journal of Orthopaedic Research, Vol. 7, No. 4, 1989, pp. 568-78. doi:10.1002/jor.1100070415
[16] H. Yuan, K. Kurashina, J. D. de Bruijn, Y. Li, K. de Groot and X. Zhang, "A Preliminary Study on Osteoinduction of Two Kinds of Calcium Phosphate Ceramics," Biomaterials, Vol. 20, No. 19, 1999, pp. 1799-1806. doi:10.1016/S0142-9612(99)00075-7

[17] S. Fujibayashi, M. Neo, H. M. Kim, T. Kokubo and T. Nakamura, "Osteoinduction of Porous Bioactive Titanium Metal," Biomaterials, Vol. 25, No. 3, 2004, pp. 443450. doi:10.1016/S0142-9612(03)00551-9

[18] T. Tanaka, M. Saito, M. Chazono, Y. Kumagae, T. Kikuchi, S. Kitasato and K. Marumo, "Effects of Alendronate on Bone Formation and Osteoclastic Resorption after Implantation of Beta-Tricalcium Phosphate," Journal of Biomedical Materials Research, Part A, Vol. 93, No. 2, 2010, pp. 469-474.

[19] M. Chazono, T. Tanaka, S. Kitasato, T. Kikuchi and K. Marumo, "Electron Microscopic Study on Bone Formation and Bioresorption after Implantation of Beta-Tricalcium Phosphate in Rabbit Models," Journal of Orthopaedic Science, Vol. 13, No. 6, 2008, pp. 550-555. doi:10.1007/s00776-008-1271-1

[20] A. Ogose, N. Kondo, N. Kudo, T. Hotta, N. Endo, H. Irie, H. Inoue and M. Ohishi, "Collagen Fibrils within Micropores of $\beta$-Tricalcium Phosphate with Cultured Cells," Japanese Society of Orthopaedic Ceramic Implants, Vol. 25, No. 1, 2005, pp. 15-18.

[21] M. C. von Doernberg, B. von Rechenberg, M. Bohner, S. Grünenfelder, G. H. van Lenthe, R. Müller, B. Gasser, R. Mathys, G. Baroud and J. Auer, "In Vivo Behavior of Calcium Phosphate Scaffolds with Four Different Pore Sizes," Biomaterials, Vol. 27, No. 30, 2006, pp. 5186-5198. doi:10.1016/j.biomaterials.2006.05.051 